\title{
Correction to: Real Hypersurfaces in the Complex Hyperbolic Quadric with Parallel Ricci Tensor
}

Gyu Jong Kim and Young Jin Suh

\section{Correction to: Result Math (2019) 74:33 https://doi.org/10.1007/s00025-019-0961-7}

In this corrigendum, we acknowledge that, due to unfortunate circumstances, several passages from Abstract and Introduction of the manuscript "Real hypersurfaces in the complex hyperbolic quadric with parallel Ricci tensor" have been taken from the original paper [8], partly with minor modifications. The correct abstract and introduction are given below.

ABstRact. In this article, the notion of parallel Ricci tensor for real hypersurfaces in the complex hyperbolic quadric $Q^{m *}=\mathrm{SO}_{m, 2}^{o} / \mathrm{SO}_{m} \mathrm{SO}_{2}$ is introduced. Moreover, real hypersurfaces in $Q^{m *}=S_{m, 2}^{o} / \mathrm{SO}_{m} \mathrm{SO}_{2}$ with Ricci parallelism are completely classified.

\section{Introduction}

As an example of Hermitian symmetric space with rank 2 of non-compact type, we can give the complex hyperbolic quadric $Q^{m *}=S_{m, 2}^{o} / S_{m} S_{2}$ (see Berndt and Suh [1]), which are known to be a kind of real Grassmann manifolds of non-compact type with rank 2 (see Besse [2] and Helgason [3]).

The original article can be found online at https://doi.org/10.1007/s00025-019-0961-7. 
Besides of the complex structure $J$ it is known that there is another distinguished geometric structure on $Q^{m *}=\mathrm{SO}_{m, 2}^{o} / \mathrm{SO}_{m} \mathrm{SO}_{2}$, namely a parallel rank two vector bundle $\mathfrak{A}$ which contains an $S^{1}$-bundle of real structures, that is, complex conjugations $A$ on the tangent spaces of $Q^{m *}$. This geometric structure determines a maximal $\mathfrak{A}$-invariant subbundle $\mathcal{Q}$ of the tangent bundle $T M$ of a real hypersurface $M$ in $Q^{m *}=S O_{m, 2}^{o} / S O_{m} S_{2}$ (see Klein and Suh [5], Suh [9]).

Accordingly, the complex hyperbolic quadric $Q^{m *}$ admits two important geometric structures, a complex conjugation structure $A$ and a Kähler structure $J$, which anti-commute with each other, that is, $A J=-J A$. Then, for $m \geq 2$, the triple $\left(Q^{m *}, J, g\right)$ is a Hermitian symmetric space of non-compact type with rank 2 and its maximal sectional curvature is equal to -4 (see Klein [4], Kobayashi and Nomizu [6], and Reckziegel [7]).

In the paper [9] due to Suh, we investigated this problem for the complex hyperbolic quadric $Q^{m *}=S O_{m, 2}^{o} / S_{m} S_{2}$ and obtained the following result:

Theorem A. Let $M$ be a real hypersurface of the complex hyperbolic quadric $Q^{m *}=S_{m, 2}^{o} / S_{m} S_{2}, m \geq 3$. The Reeb flow on $M$ is isometric if and only if $m$ is even, say $m=2 k$, and $M$ is locally congruent to an open part of a tube around a totally geodesic $\mathbb{C} H^{k} \subset Q^{2 k^{*}}$ or a horosphere whose center at infinity is $\mathfrak{A}$-isotropic singular.

If we consider a hypersurface $M$ in the complex hyperbolic quadric $Q^{m *}$, naturally two kinds of singular normal vector fields $N$ are appeared. One is said to be $\mathfrak{A}$-isotropic, and the other is $\mathfrak{A}$-principal (see $[1,9])$. In the first case where $N$ is $\mathfrak{A}$-isotropic, it is shown in Theorem A that $M$ is locally congruent to a tube over a totally geodesic complex hyperbolic space $\mathbb{C} H^{k}$ in $Q^{2 k^{*}}$ or a horosphere.

When the unit normal $N$ is $\mathfrak{A}$-principal, Berndt and Suh [1] gave a complete classification for contact hypersurfaces $M$ in $Q^{m *}$ as follows:

Theorem B. Let $M$ be a connected orientable real hypersurface with constant mean curvature in the complex hyperbolic quadric $Q^{m *}=\mathrm{SO}_{m, 2}^{o} / \mathrm{SO}_{m} \mathrm{SO}_{2}$, $m \geq 3$. Then $M$ is a contact hypersurface if and only if $M$ is congruent to an open part of one of the following contact hypersurfaces in $Q^{m *}$ :

(i) a tube of radius $r \in R^{+}$around the Hermitian symmetric space $Q^{m-1}$ * which is embedded in $Q^{m *}$ as a totally geodesic complex hypersurface;

(ii) a horosphere in $Q^{m *}$ whose center at infinity is the equivalence class of an $\mathfrak{A}$-principal geodesic in $Q^{m *}$;

(iii) a tube of radius $r \in R^{+}$around the $m$-dimensional real hyperbolic space $\mathbb{R} H^{m}$ which is embedded in $Q^{m *}$ as a real space form of $Q^{m *}$.

Motivated by the above result, in this paper we want to give a classification for real hypersurfaces in the complex hyperbolic quadric $Q^{m *}$ with Ricci parallelism $\nabla$ Ric $=0$, where Ric denotes the Ricci tensor of $M$ in $Q^{m *}$, as follows: 
Main Theorem. Let $M$ be a real Hopf hypersurface in the complex hyperbolic quadric $Q^{m *}=S_{m, 2}^{o} / S_{m} S_{2}, m \geq 4$, with parallel Ricci tensor. Then $M$ is locally congruent to one of the following

(i) a hypersurface with 3 distinct constant principal curvatures, where $\lambda$ and $\mu$ are solutions of a quadratic equation

$$
(m-2) x^{2}+\alpha x-(m-1) \alpha^{2}+2(m-2)=0,
$$

with $\mathfrak{A}$-principal unit normal and multiplicities $m-1$, respectively, and the principal curvature spaces are given by

$$
T_{\alpha}=[\xi], \phi T_{\lambda}=T_{\mu}, \operatorname{dim} T_{\lambda}=\operatorname{dim} T_{\mu}=m-1,
$$

where the Reeb function $\alpha$ is constant and $\alpha^{2}=\frac{6(m-2)}{2 m-1}$,

(ii) a hypersurface with at most 4 distinct constant principal curvatures, where $\lambda$ and $\mu$ are solutions of a quadratic equation

$$
(m-3) x^{2}+\alpha x-(m-2) \alpha^{2}+3(m-3)=0,
$$

with $\mathfrak{A}$-isotropic unit normal and multiplicities $m-2$, respectively, and the principal curvature spaces are given by

$$
T_{\alpha}=[\xi], T_{0}=[A \xi, A N], \phi T_{\lambda}=T_{\mu}, \operatorname{dim} T_{\lambda}=d i m T_{\mu}=m-2,
$$

where the Reeb function $\alpha$ is constant and $\alpha^{2} \geq \frac{12(m-3)^{2}}{(2 m-5)^{2}}$.

We want to compose our article as follows. In Sect. 2 we introduce basic geometric structures for the complex hyperbolic quadric $Q^{m *}$, which are recently developed by Klein and Suh [5]. In Sect. 3, we want to study the geometry of the maximal subbundle $\mathcal{Q}$ of $T M$ in $Q^{m *}=S_{m, 2}^{o} / S_{m} \mathrm{SO}_{2}$. Also, some equations including Codazzi and fundamental expressions related to the vector fields $\xi, N, A \xi$, and $A N$ for the complex conjugation $A$ of $M$ in $Q^{m *}$ are introduced.

In Sect. 4, we obtain the formula of Ricci tensor from the equation of Gauss for real hypersurfaces $M$ in $Q^{m *}=S O_{m, 2}^{o} / S O_{m} S O_{2}$ and get some formulas by the assumption of parallel Ricci tensor and $\mathfrak{A}$-principal normal vector field. As a remark we also show that a tube over a totally geodesic complex hyperbolic hyperquadric $Q^{m-1^{*}}$ in $Q^{m *}=S O_{m, 2}^{o} / S_{m} S_{2}$ does not admit a parallel Ricci tensor.

For a real hypersurface $M$ in the complex quadric $Q^{m}$ with parallel Ricci tensor, in order to get the main results in [8] due to the second author, we have further assumed that the unit normal $N$ is $\mathfrak{A}$-principal or $\mathfrak{A}$-isotropic, respectively. But in this paper, fortunately, in Lemma 5.4 of Sect. 5 we can derive the result that $N$ is $\mathfrak{A}$-principal or $\mathfrak{A}$-isotropic from the assumption of Ricci parallelism $\nabla$ Ric $=0$. So Lemma 5.4 gives a key role in the proof of our Main Theorem. Accordingly, by virtue of Lemma 5.4, without the assumption of singular normal vector field $N$ we can give a complete classification of our Main Theorem. 
In Sects. 6 and 7, we present the proof of Main Theorem with $\mathfrak{A}$-principal or $\mathfrak{A}$-isotropic unit normal, respectively. The first part of these proofs in Sects. 6 and 7 is devoted to give some fundamental formulas from the Ricci parallelism $\nabla$ Ric $=0$ and $\mathfrak{A}$-principal or $\mathfrak{A}$-isotropic unit normal vector field. In the latter part of each proof, related to the parallelism of the Ricci tensor $\nabla$ Ric $=0$, we will use the expression of the shape operator for real hypersurfaces in $Q^{m *}=S O_{m, 2}^{o} / S_{m} S_{2}$.

\section{References}

[1] Berndt, J., Suh, Y.J.: Contact hypersurfaces in Kaehler manifold. Proc. AMS 23, 2637-2649 (2015)

[2] Besse, A.L.: Einstein Manifolds. Springer, Berlin (2008)

[3] Helgason, S.: Geometric Analysis on Symmetric Spaces, Mathematical Survey and Monographs, vol. 39, 2nd edn. American Mathematical Society, Providence (2008)

[4] Klein, S.: Totally geodesic submanifolds in the complex quadric. Differ. Geom. Appl. 26, 79-96 (2008)

[5] Klein, S., Suh, Y.J.: Contact real hypersurfaces in the complex hyperbolic quadric. Ann. Mate. Pura Appl. https://doi.org/10.1007/s10231-019-00827-y

[6] Kobayashi, S., Nomizu, K.: Foundations of Differential Geometry. Wiley Classics Library Ed., vol. II. Wiley-Interscience Publication, Hoboken (1996)

[7] Reckziegel, H.: On the Geometry of the Complex Quadric: Geometry and Topology of Submanifolds VIII (Brussels/Nordfjordeid 1995), pp. 302-315. World Scientific Publishing, River Edge, NJ (1995)

[8] Suh, Y.J.: Real hypersurfaces in the complex quadric with parallel Ricci tensor. Adv. Math. 281, 886-905 (2015)

[9] Suh, Y.J.: Real hypersurfaces in the complex hyperbolic quadric with isometric Reeb flow. Commun. Contemp. Math. 20, 1750031 (2018)

Gyu Jong Kim

Department of Mathematics Education

Woosuk University

Wanju Jeonbuk 55338

Republic of Korea

e-mail: hb2107@naver.com 
Young Jin Suh

Department of Mathematics and RIRCM College of Natural Sciences Kyungpook National University

Daegu 41566

Republic of Korea

e-mail: yjsuh@knu.ac.kr

Publisher's Note Springer Nature remains neutral with regard to jurisdictional claims in published maps and institutional affiliations. 